\title{
The limits of agency in walking humans ${ }^{\text {is }}$
}

\author{
O.A. Kannape ${ }^{\mathrm{a}, 1}$, L. Schwabe ${ }^{\mathrm{a}, 1}, \mathrm{~T}$. Tadi ${ }^{\mathrm{a}}$, O. Blanke ${ }^{\mathrm{a}, \mathrm{b}, *}$ \\ a Laboratory of Cognitive Neuroscience, Brain Mind Institute, Swiss Federal Institute of Technology, Station 19, 1015 Lausanne, Switzerland \\ b Department of Neurology, University Hospital, 1211 Geneva 14, Switzerland
}

\section{A R T I C L E I N F O}

\section{Article history:}

Received 9 November 2009

Received in revised form 28 January 2010

Accepted 1 February 2010

Available online 8 February 2010

\section{Keywords:}

Motor awareness

Self

Virtual reality

Motion capture

Sensorimotor

Sleepwalking

\begin{abstract}
A B S T R A C T
An important principle of human ethics is that individuals are not responsible for actions performed when unconscious. Recent research found that the generation of an action and the building of a conscious experience of that action (agency) are distinct processes and crucial mechanisms for self-consciousness. Yet, previous agency studies have focussed on actions of a finger or hand. Here, we investigate how agents consciously monitor actions of the entire body in space during locomotion. This was motivated by previous work revealing that (1) a fundamental aspect of self-consciousness concerns a single and coherent representation of the entire spatially situated body and (2) clinical instances of human behaviour without consciousness occur in rare neurological conditions such as sleepwalking or epileptic nocturnal wandering. Merging techniques from virtual reality, full-body tracking, and cognitive science of conscious action monitoring, we report experimental data about consciousness during locomotion in healthy participants. We find that agents consciously monitor the location of their entire body and its locomotion only with low precision and report that while precision remains low it can be systematically modulated in several experimental conditions. This shows that conscious action monitoring in locomoting agents can be studied in a fine-grained manner. We argue that the study of the mechanisms of agency for a person's full body may help to refine our scientific criteria of selfhood and discuss sleepwalking and related conditions as alterations in neural systems encoding motor awareness in walking humans.
\end{abstract}

(c) 2010 Elsevier Ltd. All rights reserved.

\section{Introduction}

The generation of a goal-directed action and the building of a conscious experience thereof have been considered distinct processes (Castiello, Paulignan, \& Jeannerod, 1991) and science has provided empirical evidence for such dissociations in neurological patients. Seminal hand-pointing studies have revealed that patients, who cannot visually recognize objects or shapes, can nevertheless accurately direct and orient their hands when reaching towards such objects or shapes (Goodale, Milner, Jakobson, \& Carey, 1991); such instances of motor responses to stimuli without awareness have also been demonstrated in healthy participants (Bridgemen, Kirch, \& Sperling, 1981; Goodale, Pelisson, \& Prablanc, 1986).

Being aware of the goal of an action or "what" an action is about is not the same as being aware of "how" that action was

\footnotetext{
is Disclaimer: The research presented in this manuscript has not been previously published nor has it been submitted simultaneously for publication elsewhere.

* Corresponding author at: Laboratory of Cognitive Neuroscience, Brain-Mind Institute, Station 19, Ecole Polytechnique Fédérale de Lausanne (EPFL), 1015 Lausanne, Switzerland. Tel.: +41216939621; fax: +41216939625.

E-mail address: olaf.blanke@epfl.ch (O. Blanke).

1 These authors contributed equally to this work.
}

performed. Following early work by Nielsen $(1963,1978)$, this latter form of conscious action monitoring or motor awareness (MA) has recently been the topic of intensive research (Fourneret \& Jeannerod, 1998; Franck et al., 2001; Knoblich \& Kircher, 2004; van den Bos \& Jeannerod, 2002). In these studies participants' MA was measured in response to randomized incongruencies between generally congruent visual, proprioceptive, and motor signals. These studies showed that participants automatically aligned their hand trajectories with a visual target on the computer screen, while compensating for a displayed spatial deviation. The participants were often unaware of their online corrections and judged many of these actions as non-deviated.

These previous investigations of MA were important for the study of bodily self-consciousness, but were focussed on the awareness for actions of fingers, hands, or arms. Yet, a fundamental aspect of bodily self-consciousness is that the self is experienced as a single coherent representation of the entire, spatially situated body, not as several separate body parts (Blanke \& Metzinger, 2009) and this has recently been tested experimentally (Aspell, Lenggenhager, \& Blanke, 2009; Ehrsson, 2007; Lenggenhager, Tadi, Metzinger, \& Blanke, 2007). Therefore, previous MA studies did not investigate this fundamental aspect of the bodily self as the participants' body position was kept constant (except for actions of finger, hand, or arm). To investigate MA for the entire body we here measured 
MA during different displacements of a person's entire body during locomotion. In addition to differences in the involved sensorimotor systems, locomotion also gives rise to vestibular sensations and changes in the perception of the surrounding extrapersonal space, differing from changes during goal-directed arm movements in stable or sitting actors. Striking dissociations between MA and motor performance (MP) for the full body, are suggested by anecdotal clinical reports about patients with episodic nocturnal wandering (as in nocturnal frontal lobe epilepsies; Pedley \& Guilleminault, 1977; Plazzi, Tinuper, Montagna, Provini, \& Lugaresi, 1995) or sleep walking (somnambulism; Broughton, 1968; Gastaut \& Broughton, 1965). These patients may get up and walk around their house for several minutes without consciously monitoring or being able to recall what and how it happened (Broughton, 1968; Plazzi et al., 1995). This lack of MA for the entire body leads to distinct clinical, scientific, and legal consequences for such patients and is apparent in medical and forensic procedures (Beran, 2002; Mahowald, Bundlie, Hurwitz, \& Schenck, 1990).

Merging techniques from virtual reality, full-body tracking, and cognitive science we investigated MA and MP for a person's entire body during locomotion (Fig. 1a). Participants' movements were captured (participant's body, Fig. 1a) and projected in real-time onto a virtual body that mimicked the participant's movements (virtual body, Fig. 1b) while they performed a goal-directed walking task. During some trials the virtual body's walking trajectory was deviated systematically from the participant's actual walking trajectory allowing us to generate controlled spatial conflicts and to experimentally test MA of healthy participants during locomotion. In study 1 we tested - based on visual-motor conflicts - whether MA and MP for the full-body is dependent on the angle of the deviated walking trajectory. In study 2 , we tested (a) whether MP and MA (as tested in study 1) may be further influenced by the orientation (upright, inverted; i.e. Reed, Stone, Bozova, \& Tanaka, 2003) and the perspective or walking direction (congruent, incongruent; i.e. David et al., 2006; Vogeley \& Fink, 2003) as both mechanisms, inversion and perspective, have shown to influence the perception of human bodies as well as bodily self-consciousness (see also below, study 2 ).

\section{Materials and methods}

\subsection{Participants}

Nine healthy participants volunteered for study 1 (6 male, 3 female, mean age $=21 \pm 3$ years), 14 for study 2 ( 8 male, 6 female, mean age $=25 \pm 6$ years, height $=176 \pm 10 \mathrm{~cm}$, weight $=69 \pm 12 \mathrm{~kg}$ ). Participants had normal or corrected to normal vision. The two studies were conducted according to the principles expressed in the Declaration of Helsinki and accepted by the local ethics committee (University Hospital, Lausanne, Switzerland). All participants provided written informed consent for the collection of data and subsequent analysis.

\subsection{Motion capture}

An active optical motion capture system was used for tracking and recording the participants' movements (ReActor2, Ascension Technology Corp., Burlington, VT, USA). In both studies 20 infra-red markers were strategically placed on the participants' body and major joints. Whole-body motion capture took place in a $4.11 \mathrm{~m} \times 4.11 \mathrm{~m} \times 2.54 \mathrm{~m}$ (length $\times$ width $\times$ height) tracking arena incorporating 448 detectors positioned in the 12 bar tracking frame. Motion was captured at a sampling frequency of $30 \mathrm{~Hz}$.

\subsection{Character animation}

For 3D character animation a commercial, real-time productivity suite was used (Autodesk MotionBuilder, San Rafael, CA, USA). This software facilitated mapping the virtual character used in the study onto the set of optical markers worn by the participant while integrating natural biped kinematics. A customized skeleton was fitted for each participant to align the joints of the virtual character with the participant's actual joints. The overall delay of the system, including data acquisition, character animation, and visual presentation, was less than $80 \mathrm{~ms}$.

\subsection{Visual stimuli}

Visual stimuli were back-projected onto an area of $3.20 \mathrm{~m} \times 2.35 \mathrm{~m}$ (width $\times$ height, $1280 \times 1024$ pixels, $60 \mathrm{~Hz}$ ), with the screen itself forming the back-wall of the tracking arena (JVC DLA-SX21 projector, JVC U.S.A., Wayne, NJ, USA) The dimensions of the virtual room matched those of the tracking arena. All cameras were adapted (location, field-of-view) so the virtual body had the same proportions as an actual person standing at the same distance. A custom plug-in controlled the experiment flow, formats, and saved the position-, timing-, and response data during the entire study.

\subsection{Study 1: Procedure}

At the beginning of the study, participants were given three minutes to adapt to their virtual body in a "free movement" condition. The virtual room was empty except for the participant's virtual body. At this point the participants also had the opportunity to point out any perceived mismatches between his own and the virtual body's movements so that these could be corrected. A training block (20 trials, 5-10 min) was run before each of the four main conditions. The same targets that were used in the main experiment were used here as well, but only angular deviations of $\pm 5^{\circ}$ and $\pm 20^{\circ}$. During the training session the participant could ask questions about the task and was advised to give constant feedback about his movement and decision.

In each trial of study 1 , participants first moved the virtual body to the start location in the virtual room by walking to the corresponding location in the motion capture area. Then, a virtual target was shown in the virtual room. Its location was randomly selected from four possible locations and indicated by a transparent cylinder (Fig. 1). After the virtual target appeared, the participants were asked to move their virtual body to the virtual target location by walking in the motion capture area. In some trials (75\%), in randomized order, and beyond a distance of $30 \mathrm{~cm}$ from the start location, the walking trajectory of the virtual body was systematically deviated towards either the left or the right (by $5^{\circ}, 10^{\circ}$, $15^{\circ}$, or $30^{\circ}$ ). The deviation of the virtual body was calculated relative to the straight line between the participants' current position and the position of deviation onset. Participants were prevented from adapting to these angular deviations as their direction and amplitude was randomized on a trial-by-trial basis in study 1 and 2. We predicted that participants would spontaneously deviate from the straight walking trajectory that connected the start and target location in order to move the virtual body to the virtual target location, as has been shown previously for goal-directed hand movements (Fourneret \& Jeannerod, 1998; Nielsen, 1963). A trial ended with the target cylinder disappearing as soon as the participant reached the target distance of $180 \mathrm{~cm}$, independent of his/her final position with respect to the virtual target. Each participant was explicitly instructed to always try to reach the target with their virtual body as accurately as possible. The exact instructions were: "Move your virtual body directly into the target cylinder, in one, 

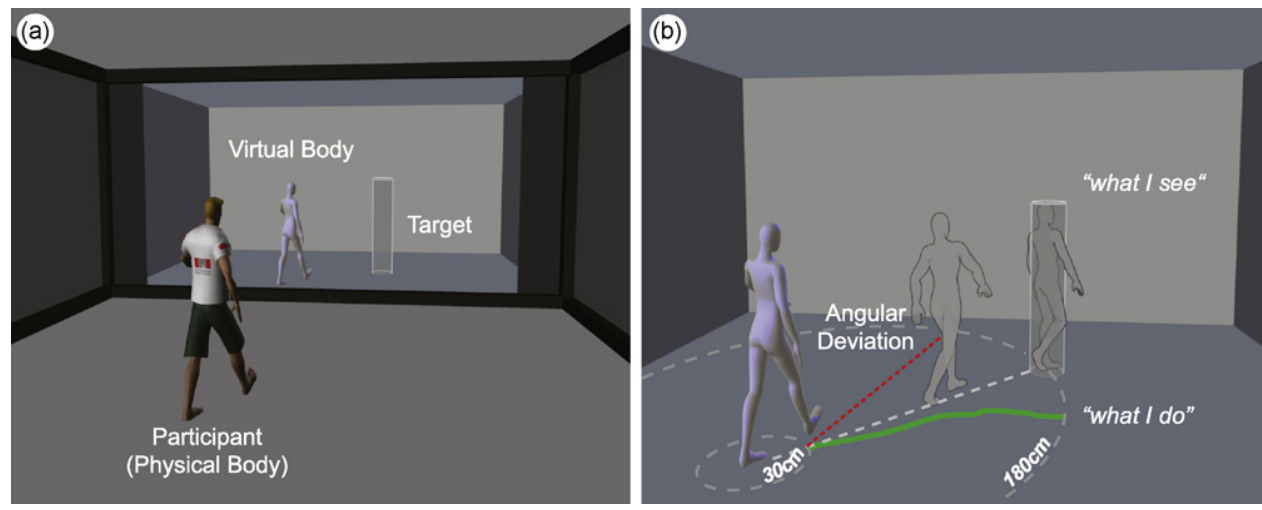

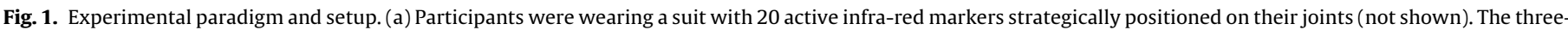

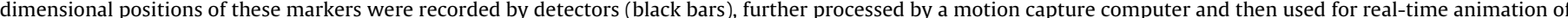

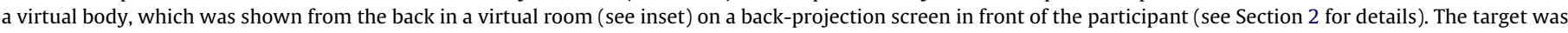

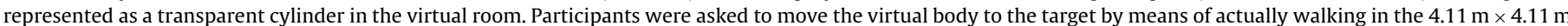

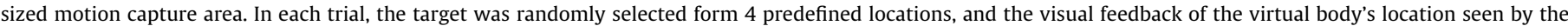

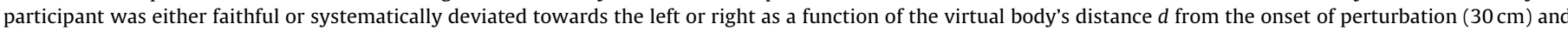

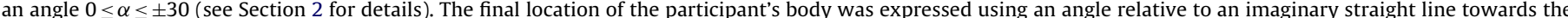

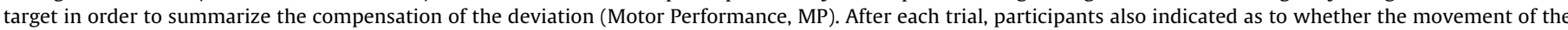

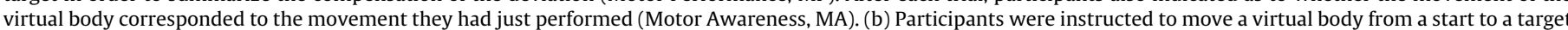

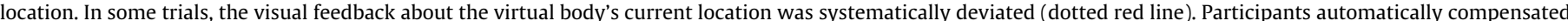
(solid green line) from a straight line in order to move the virtual body into the target location (dashed gray line).

smooth movement. Once you reach the cylinder it will turn off, and a question is displayed on the screen saying: 'Did the movement shown on the screen correspond to the movement you just performed?"' The participant was advised to include the movement per se ( $\sim$ kinematics), its direction, and its speed into his/her decision. A wireless Microsoft XBOX 360 controller was used by the participant to start each trial and give the yes/no response.

\subsection{Analysis}

For all experimental conditions we determined three values (as explained in detail in the following paragraphs). First, we measured the participants' walking trajectories and movement endpoints to quantify MP. Second, we asked participants to indicate their MA by specifying after each trial whether the movement shown on the wall of the motion capture area corresponded to the movement they had just performed (Franck et al., 2001). Third, the relationship between MP (movement endpoint) and MA was quantified for all experimental conditions by calculating a motor awareness index (MAI). This index indicated the likelihood of committing an error in MA with respect to the actual MP.

\subsection{Motor performance}

MP described the total angle compensated by the participant taking into account the endpoint of each of their movement trajectories and was measured from the onset of deviation at a distance of $30 \mathrm{~cm}$ to the start location and measured in degree. The mean position of the four hip markers was used to analyse all walking trajectories. Trials that were longer than $10 \mathrm{~s}$ in duration and trials that were corrupted through marker occlusions were omitted. A mean trajectory was obtained by taking the arithmetic mean of the $x$ and $z$ coordinates at each sample across all trials with the same angular deviation.

\subsection{Motor awareness}

MA was expressed by the number of yes-responses out of all valid trials. Correct MA or self-attribution was a "yes" response for undeviated, a "no" response for deviated trials. For analysis by itself, the $50 \%$ threshold values (point of subjective ambiguity) were calculated using the Matlab Psignifit toolbox; with respect to the Motor Awareness Index, MA was expressed as the percentage of incorrectly self-attributed, deviated trials.

\subsection{Motor awareness index}

The MAI was introduced to describe the likelihood of errors in MA (\%self-attribution) with respect to the gain (MP/angular deviation) for each participant and each condition with an angular deviation. High values of the MAI reflected that participants were more likely to misattribute the full body action to themselves (caused by frequent self-attribution errors, a low gain, or a combination of both), whereas low values indicated participants were less likely to self-attribute these actions. A high MAI would indicate that MP and MA may be distinct processes because even a strong sensorimotor conflict (caused by compensating for larger angular deviations) would not cause participants to not self-attribute. The MAI was therefore used as a mean to investigate the relationship between MA and MP and how this changed across different experimental conditions. Thus, the MAI allowed us to determine how the otherwise inaccessible relationship between the initially presented angular deviation and the actual motor correction influenced MA.

The MAI was calculated by taking the ratio of MA and MP for each deviation angle. MA was expressed in terms of percent false self-attributions, whereas in order to compare MP to MA, MP was re-calculated as the relative angular compensation performed based on the introduced angular deviation. The $0^{\circ}$ control trials were hence omitted in this case (please refer to the supplementary material for further details on MP, MA, and MAI).

\subsection{Study 2: Procedure}

Motion capture, stimulus animation, procedure, training, and analysis in study 2 were the same as in study 1 . In study 2 , we examined whether bodily constraints such as the congruency between the orientation and walking direction of the participant's body and the orientation and walking direction of the virtual body may alter full-body MP, MA, and/or MAI (see also below). Orientation and direction mechanisms were tested by manipulating the orientation (depicting the virtual body either upright [as in study 1] or inverted) and the direction (depicting the virtual body either in back-view [as 

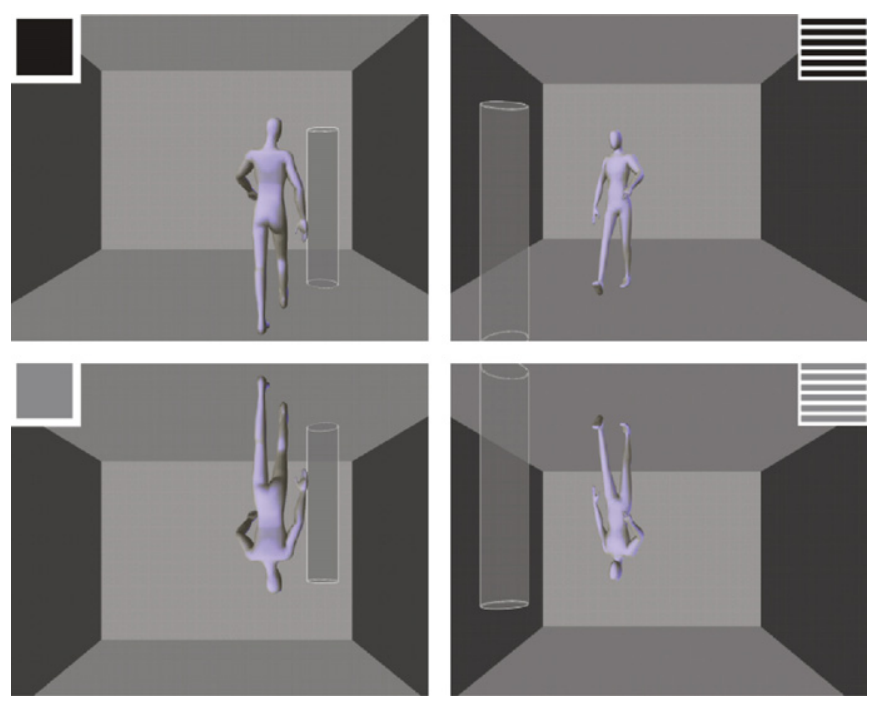

Fig. 2. Visual feedback for the four conditions. Examples of the visual feedback shown to the walking participants on the back-projection screen. In the second study the virtual body would either be shown upright or inverted. It could further be shown from the back in congruence with the participant's actual walking direction, or from the front. In the latter case the virtual body appeared similar to a mirror image of the real body heading towards the participant.

in study 1] or in front-view) of the virtual body (Fig. 2) and retesting the same eight angular deviations as in study 1 (we averaged performance across the corresponding right and left deviations). This gave rise to a $2 \times 2 \times 4$ factorial design with the factors "direction", "orientation", and "angular deviations" (4 conditions with deviations). If MA decreases for front-views this would suggest that directional incongruency between the virtual body and the participant's body is important in full-body MA as tested here whereas better performance for front-view conditions would suggest that mirror expertise may be important in full-body MA. Concerning orientation and based on previous data on the so-called body inversion effect (Reed et al., 2003), we predicted that full-body MA would decrease for inverted bodies. In order to ascertain that there were no perceptual differences between the different experimental conditions the average distance (and size) of the approaching virtual body in the front-view condition was designed to be equal to the distance in the back-view condition (Fig. 2).

There were four main blocks in study 2 which were randomized for each participant, one condition per block. A main block consisted of 88 trials with 8 different angular deviations $\left( \pm 5^{\circ}, \pm 10^{\circ}\right.$, $\pm 15^{\circ}, \pm 30^{\circ}$ ) and the $0^{\circ}$ control trials (25-35 min). Each deviation was used twice at each target resulting in 64 offset trials and 24 true $\left(0^{\circ}\right.$ offset) trials. (In study 1 , only one main block was run with a total of 128 trials of which 96 were deviated, each deviation occurred three times at each target, 35-45 min.)

\section{Results}

\subsection{Study 1}

\subsubsection{Motor performance}

Fig. 3a shows the walking trajectories of one participant (in top-view) in the motion capture area from the start location to each of the four target locations in trials without angular deviation. All participants showed comparable performance and were able to accurately move the virtual body to the virtual target locations (error: $0.10^{\circ} \pm 0.31^{\circ}$, mean $\pm S E M$ ). Correct MA was high and participants judged the seen movements of the virtual body as the movements that they had performed in $92.0( \pm 2.5) \%$ of trials. However, in trials with angular deviations, and as reported for goal-directed hand movements (Fourneret, Franck, Slachevsky, \& Jeannerod, 2001; Fourneret \& Jeannerod, 1998; Nielsen, 1963), our experimental design led participants to change their walking direction (Fig. 3b): walking trajectories were deviated in the direction opposite to the direction of the angular deviation moving the virtual body towards the virtual target. For subsequent analysis we rotated each trajectory by the corresponding angle of the target and averaged the walking trajectories across the four virtual target locations (Fig. 3c). Fig. 3d illustrates the negative correlation between the endpoints of the participants' walking trajectories and the size of the angular deviation added to the virtual body's walking trajectory $\left(r^{2}=0.995, p<0.001\right)$. The average gain of MP was 0.41 $( \pm 0.07))$.

\subsubsection{Motor awareness}

How is this automatic compensation for the angular deviations reflected in the participants' motor awareness? MP and MA are summarized in Fig. 4a showing that errors in MA were common for trials with small angular deviations. These deviations of $5^{\circ}, 10^{\circ}$, and $15^{\circ}$ lead to many erroneous self-attributions (in $29.2-82.9 \%$ of all trials) decreasing in magnitude with increasing angular deviation. Thus, participants often judged the seen movements of the virtual body as the movements they had just performed. Such errors were rare (or absent in some participants) when the angular deviations were largest (5.6\%). The point of subjective ambiguity, that is, the point at which participants were equally likely to judge the movement of the seen virtual body as the movement they just performed or not, corresponded to $9.6^{\circ}$. In other words, the majority of deviated trials below this threshold were judged by participants not to be deviated indicating that motor control for full body displacements may operate with decreased and low MA in these conditions.

\subsubsection{Motor awareness index}

Fig. $4 \mathrm{~b}$ illustrates the MAI across all eight tested angular deviation and shows that the highest value was at $\pm 5^{\circ}$ and $\pm 10^{\circ}$ corroborating our data on the subjective ambiguity (Fig. 4b) and suggesting that - as the gain was constant at all tested angular deviations -the more frequent errors in MA are not due to a lower gain in MP at angular deviations above $10^{\circ}$.

\subsection{Study 2}

MP and MA as tested in study 1 may be influenced in the highly trained processing of upright bodies, and it may not transfer to less trained postures such as inverted bodies that have been shown to decrease performance in the perception of body postures (Reed et al., 2003) and biological motion (Cutting, Moore, \& Morrison, 1988), as well as self-attribution of arm actions (Knoblich \& Kircher, 2004; Knoblich, Stottmeister, \& Kircher, 2004). Moreover, performance may also be dependent on the perspective (David et al., 2006; Vogeley \& Fink, 2003) or direction from which the virtual body is shown as MA during goal-directed arm movements has been shown to decrease if the direction of the virtual arm movement is rotated by $180^{\circ}$ (van den Bos \& Jeannerod, 2002). Similar directional mechanisms have been reported for body ownership (Tsakiris \& Haggard, 2005). Concerning the full body, it has also been shown that self-identification and self-location can be manipulated by visuo-tactile stimulation from a back-view direction (Ehrsson, 2007; Lenggenhager et al., 2007), although humans are more used to seeing themselves in front-view due to the common use of mirrors.

\subsubsection{Motor performance}

Fig. 5 illustrates MP across all angular deviations for the four experimental conditions. Our results showed a significant orientation $\times$ direction interaction $(F(1,12)=7.8682, p=0.016)$, without 
(a)

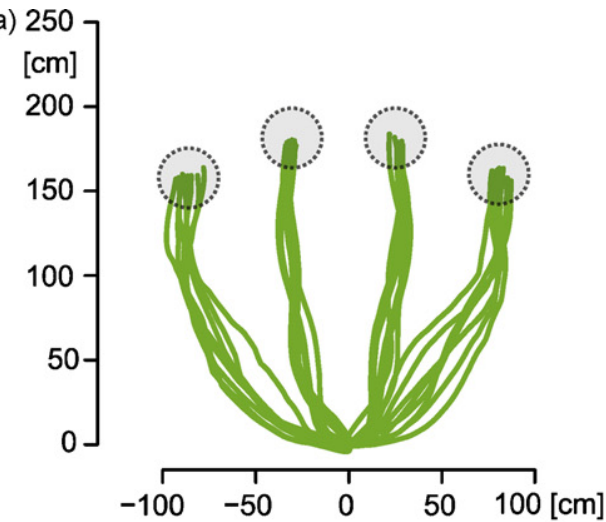

(b) 250

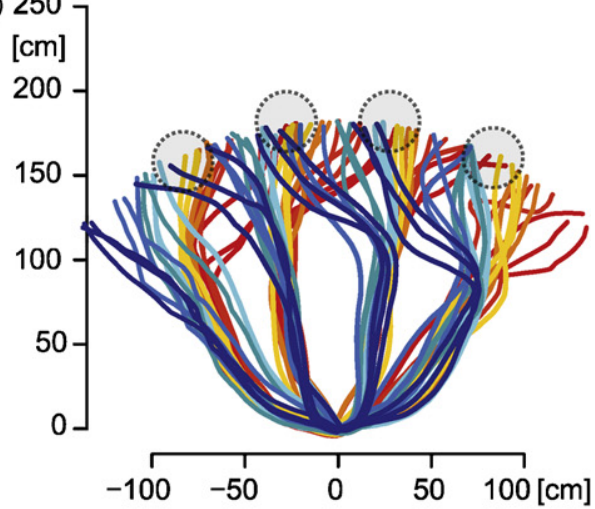

(c) 250
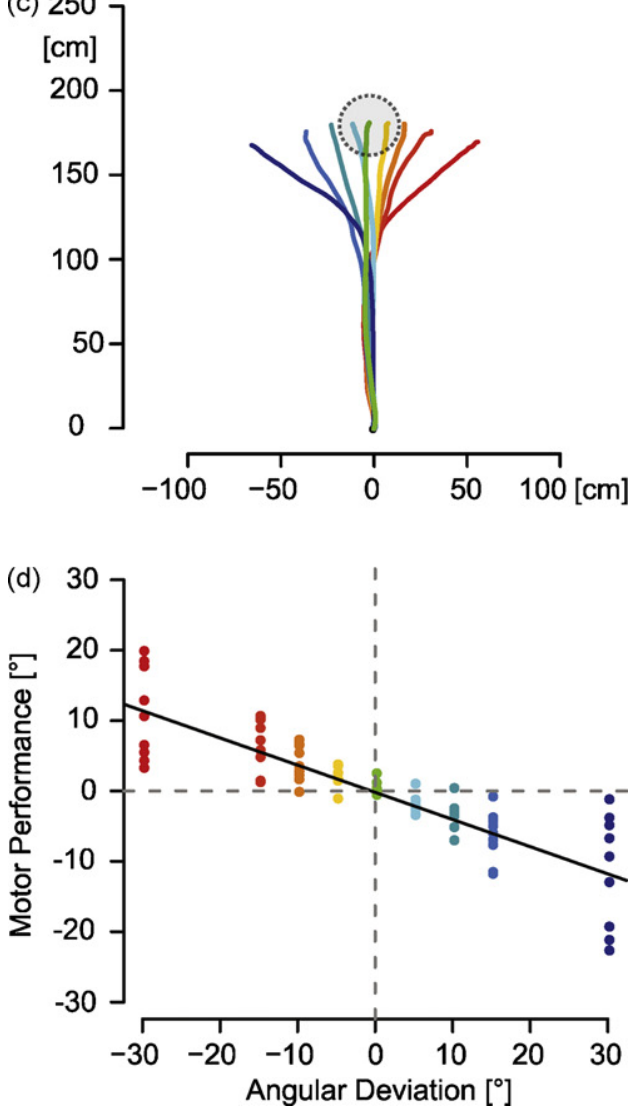

Fig. 3. Motor Performance. Walking Trajectories (single participant) and Motor Performance (all participants). (a) Walking trajectories from the start location towards the 4 predefined target locations in the trials without deviations. The gray circles indicate the target locations. (b) Walking trajectories towards the targets in the trials with deviations of the virtual body location to the left (red lines) and right significant main effects for Orientation $(F(1,12)=4.1647, p=0.06)$ or Direction $(F(1,12)=1.4963, p=0.24$; Supplementary Table 1$)$. Further statistical analysis revealed that MP was highest in the "upright/back" condition differing from MP in the "upright/front" $(p=0.01)$ and "inverted/back" conditions $(p<0.01)$ ("upright/back" versus "inverted/front" approached significance: $p=0.06$ ) (Fig. 6a). MP $\left(-0.33^{\circ} \pm 0.31^{\circ}\right)$ and MA $(96.4 \% \pm 1.42 \%)$ in undeviated trials were comparable between study 1 and 2 (Fig. 6b).

\subsubsection{Motor awareness}

In study 2, the point of subjective ambiguity for MA in the "upright/back" condition was $13.8^{\circ}\left( \pm 0.83^{\circ}\right)$. This was somewhat higher than in study 1 and lower than in the remaining three conditions (15.1-15.8 ${ }^{\circ}$. There were no differences in MA across conditions (all $p>0.18$; supplementary Table 2 ).

\subsubsection{Motor awareness index}

Analysis of the MAI revealed that participants were significantly more likely to make self-attribution errors in the inverted conditions than in the upright ones (main effect of Orientation: $F(1,12)=7.7768 ; p=0.016$; Fig. 6 c; supplementary Table 3 ; post hoc analysis revealed significant differences between "upright/back" "inverted/back" and "upright/back" - and "inverted/front" conditions: both $p<0.018$ (all other $p>0.15$ ); supplementary Table 3 ). This means larger sensorimotor mismatches, induced by the participant's automatic compensatory movement, were required in the inverted conditions in order for participants to become conscious of the mismatch and adapt their movements. In other words, the number of errors in MA was higher in the inverted conditions than in the upright conditions for the same MP and the same sensorimotor mismatch.

\section{Discussion}

With the use of virtual reality technology, full-body tracking, and sensorimotor conflicts, we induced changes in MP and MA of moving agents. This enabled us to quantify the precision with which an agent monitors the displacements of his body in extrapersonal space. Our findings show that humans track the position and locomotion of their body with a surprisingly low accuracy within a peripersonal space of $10-15^{\circ}$. This incorrect awareness during goal-directed locomotion and navigation - found under the present experimental conditions - suggests that the conscious monitoring of an agent's moving body is not reliable and that the generation of full-body locomotion and the building of a conscious experience of it are distinct brain processes.

The value of $10-15^{\circ}$ above which angular biases during locomotion were more often correctly perceived than not by our participants is compatible with earlier work on conscious monitoring of goal-directed hand and arm movements (thresholds of $6.5-15^{\circ}$ ) (Farrer, Franck, Paillard, \& Jeannerod, 2003; Franck et al., 2001; Jeannerod \& Pacherie, 2004; Posada, Franck, Augier, Georgieff, \& Jeannerod, 2007; Slachevsky et al., 2001; Synofzik, Thier, \& Lindner, 2006), as is our finding that correct MA (in trials without angular deviation and in trials with the largest angular deviations) was high. Our MAI analysis further suggests that the frequent MA errors are independent from the gain of MP (Fig. 4b). Importantly, full-body locomotion displaces the entire body of the

(blue lines) for $\pm 30^{\circ}$ (red/dark-blue), $\pm 15^{\circ}$ (dark-orange/blue), $\quad \pm 10$ (orange/turquoise) and $\pm 5^{\circ}$ (yellow/light-blue). (c) Average of all walking trajectories of this participant (using the same convention as in (a and b)) after having aligned the individual trajectories towards their respective target location. (d) Motor Performance for all participants and all 9 angles. Participants did not compensate entirely for the introduced deviation (gain: 0.41) and the compensation correlates with the deviation. 

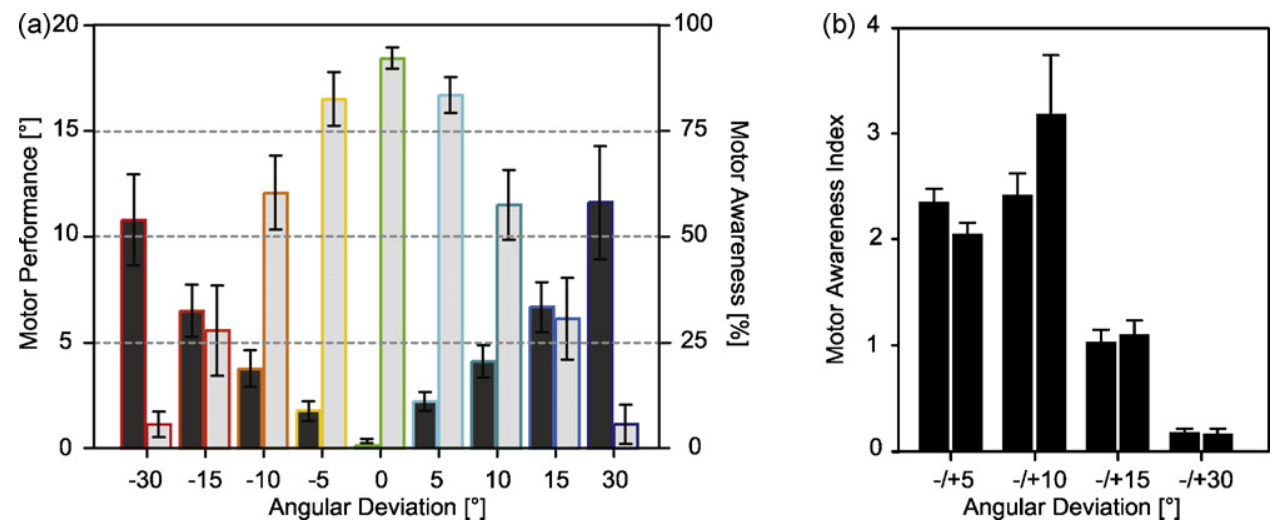

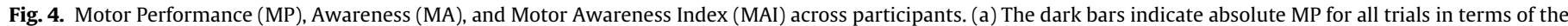

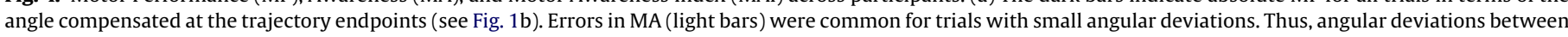

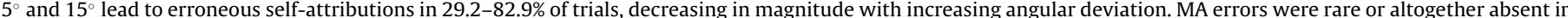

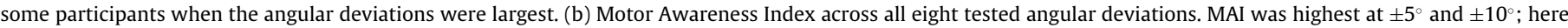

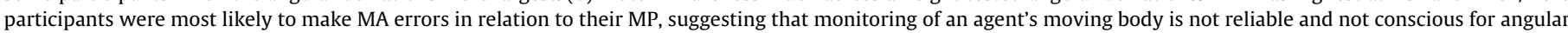
deviations below $10^{\circ}$ (all error bars are SEM).

person (and the arm and hand as well) giving rise to vestibular sensations and changes in the perception of the surrounding extrapersonal space, in addition to changes in the leg sensorimotor system. This differs from goal-directed arm movements in stable or sitting actors, where only arm related sensorimotor changes occur. Despite the different functional consequences of the movement of a person's body part or a person's entire body, the present experimental data suggest that humans rely on comparable mechanisms for monitoring the action of a single body part (i.e. arm) and the entire body. Our study therefore extended research on MA to moving agents using a goal-directed locomotion paradigm which enabled us to create experimental breaches between a virtual fullbody movement and a person's actual full-body movement. The present walking characteristics (such as average and maximum trajectory deviation) were comparable to those found in more natural experimental settings where walking performance is generally not tested under conditions with angular deviations (Hicheur, Pham, Arechavaleta, Laumond, \& Berthoz, 2007). By investigating the effects of angular deviations and especially MA measures during human locomotion we also extended and utilized techniques that have previously been used in virtual reality research for methodological purposes (Razzaque et al., 2001) to the investigation of self-consciousness in moving human agents.

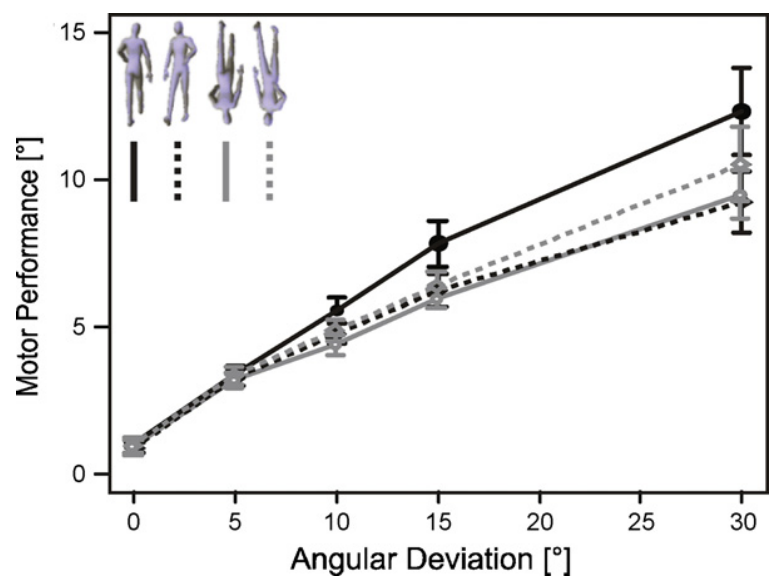

Fig. 5. Motor Performance across conditions and deviations. Motor Performance (MP) is shown for all angular deviations and experimental conditions as indicated by the small avatars in the top left corner. Participants MP was highest in the "upright back" condition (solid black line) for all tested deviations (error bars indicate the SEM). (a)
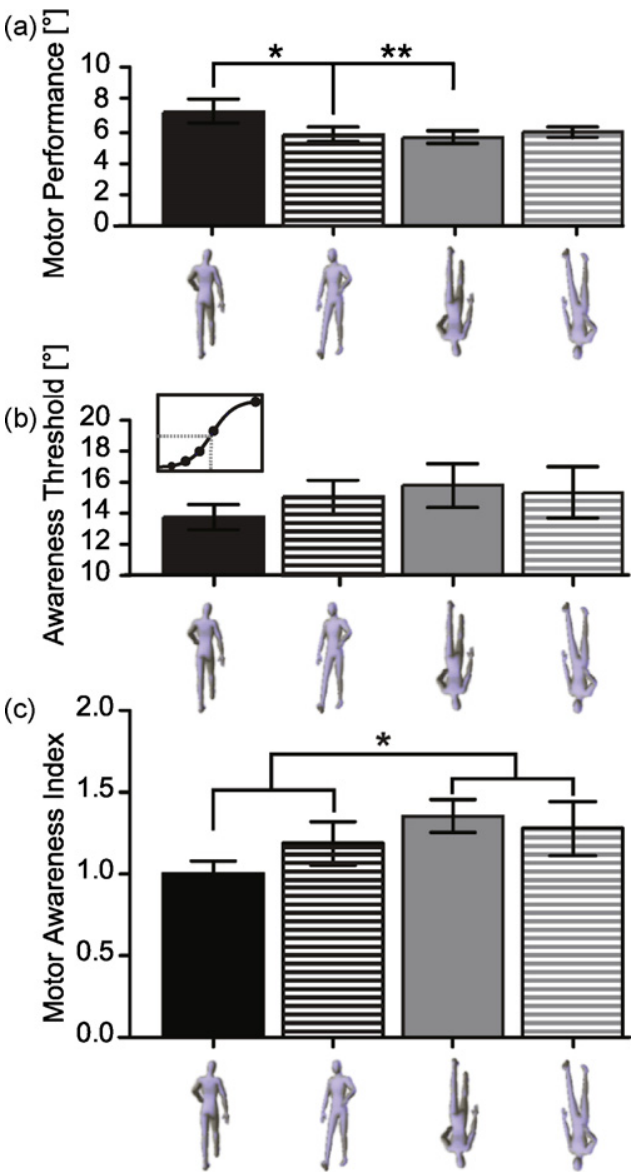

Fig. 6. Motor Performance and Motor Awareness in Study 2. (a) MP (now averaged across all tested angles per condition) was significantly higher in the "upright back" condition (black bar) than in the "upright front" condition (bar with black stripes) and the "inverted back" condition (grey bar). (b) Motor Awareness (MA) thresholds were lower in the "upright back" condition (black bar) as compared to the other conditions, but this difference did not reach significance. (c) The Motor Awareness Index (MAI) illustrates that participants were least likely to make MA errors depending on their MP in the "upright back" condition. We also note that incongruent orientation (between physical and virtual body) is reflected in the MAI. Thus, participants were significantly less likely to make MA errors in the upright conditions (black bars) than in the inverted conditions (grey bars) (all error bars are SEM). 
That MP and MA for movements of the entire body of an agent in space are in principle dissociable is suggested by rare and striking neurological syndromes such as somnambulism (Broughton, 1968; Gastaut \& Broughton, 1965) and epileptic episodic nocturnal wandering (Pedley \& Guilleminault, 1977; Plazzi et al., 1995), but has to our knowledge never been tested experimentally and never in healthy participants. An interesting aspect of these neurological syndromes is the complexity of the behaviours that are carried out while patients are unaware of what and how they performed them. In addition, such patients may be steering their body through a fairly complex environment. Complexity of movement and locomotion in humans are often regarded as indicators to consider an agent as conscious and are key constituents of selfhood clinically, philosophically, and legally (Beran, 2002; Broughton, 1968; Mahowald et al., 1990; Pedley \& Guilleminault, 1977; Revonsuo et al., 2000). Conscious human agents have been contrasted with the "zombie" of philosophy and neuropsychology (Koch \& Crick, 2001; Revonsuo et al., 2000), which is often defined as an agent who moves through the environment without conscious control. We note that such a lack of conscious control during locomotion is a crucial difference with respect to the lack of conscious control for arm or hand actions (Fourneret \& Jeannerod, 1998; Franck et al., 2001; Nielsen, 1963). In this latter case awareness is lacking for the movement of a certain body part of the agent, but the agent remains conscious of the position and locomotion of his entire body and hence preserves selfhood. The present procedure allowed us to manipulate selfhood and showed that the central mechanisms of MA for an agent's body parts and the entire agent's body are similar, despite their different functional consequences concerning selfhood (Blanke \& Metzinger, 2009).

Anecdotal neuroimaging observations in patients with sleepwalking (Broughton, 1968; Gastaut \& Broughton, 1965) and nocturnal frontal lobe epilepsy (without deambulation; Pedley \& Guilleminault, 1977; Plazzi et al., 1995) implicated the medial prefrontal cortex (including supplementary motor area and the anterior cingulate cortex) in the lack of conscious control during locomotion in humans. Unfortunately, the neuroscience and neuroimaging of upright gait is hampered by two main caveats. First, neuroimaging using fMRI, MEG, or EEG is currently not available or severely limited in walking humans, although a few research groups have employed SPECT (Fukuyama et al., 1997) and NIRS (Miyai et al., 2001; Suzuki et al., 2004). These researchers reported an involvement of medial prefrontal cortex, primary sensorimotor cortex as well as lateral premotor and prefrontal cortex during walking in humans. Second, only humans are truly upright walking primates. The neuroscience of walking is thus almost entirely based on findings in quadrupeds and behavioural work in patients with gait disorders pointing to a distributed network including a large network of cortical regions, such as the sensorimotor cortex, premotor cortex, and posterior parietal cortex, but mostly subcortical regions such as spinal cord, brainstem, basal ganglia, cerebellum (Armstrong, 1988; Drew, Prentice, \& Schepens, 2004; Grillner \& Wallen, 1985; Nutt, Marsden, \& Thompson, 1993). The sparse clinical data and neuroimaging data point towards the medial walls of prefrontal and premotor cortex in MA and MP in locomotion, but this important question remains to be studied experimentally.

Study 2 confirmed our findings of study 1 in a different participant sample by showing that MA for the full body depends on angular deviation. In study 2 we tested whether MP and MA are influenced by the highly trained processing of upright bodies and whether these effects transfer to less trained postures such as inverted bodies. Previous work has shown that inverted bodies decrease performance in the perception of body postures (Reed et al., 2003) and biological motion (Cutting et al., 1988), as well as in the self-attribution of arm actions (Knoblich \& Kircher, 2004;
Knoblich et al., 2004). Secondly, we tested whether performance also dependent on the perspective (David et al., 2006; Vogeley \& Fink, 2003) or direction from which the virtual body is shown during locomotion. Again, previous work had shown that MA for goal-directed arm movements decreases if the direction of the shown virtual arm movement (shown during movement execution via a computer screen) is rotated by $180^{\circ}$ with respect to the unseen goal-directed arm movement (van den Bos \& Jeannerod, 2002). Similar directional mechanisms have also been reported for the related sense of body ownership for the upper extremity (Tsakiris \& Haggard, 2005). Directional visuo-motor incongruencies thus seem to impair MA during goal-directed arm movements. Yet, this may be different for full body movements, as humans are more used to seeing themselves in front-view (directional incongruency) due to the common use of mirrors and not in back-view (directional congruency). Thus, in study 2 we manipulated the spatial relationship between the participant's body and the virtual body in order to investigate the role of direction and orientation on MP, MA, and MAI.

Our data show that while MA was not directly influenced by orientation and direction MP was influenced, as specifically characterized by highest gains in the upright-back condition. MAI analysis revealed that the actual angular displacement that resulted from the MP in each experimental condition (during locomotion) did affect MA. Yet, such effects on MA were only observed for inverted virtual bodies leading to higher MAI values. The present data show that larger sensorimotor mismatches were necessary in the inverted conditions before participants became aware of them. The number of errors in MA was higher in the inverted conditions than in the upright conditions, although MP was similar. These results were not reflected in MA thresholds as these did not take into account the influence of the ongoing MP. Thus, through incongruent orientation or inversion between the participant's body and the virtual body we can induce further decreases in MA (reflected in an increased MAI). This suggests that the perception of one's own full body movement under the present experimental conditions depends on orientation as has been shown for the perception of body postures (Reed et al., 2003), biological motion (Cutting et al., 1988), and arm actions (Knoblich \& Kircher, 2004; Knoblich et al., 2004). Incongruency per se (between the participant's body and the virtual body) does not lead to decreases in MA, as directional incongruency was not associated with significant decreases in MA. The similar performance between back-view and front-view virtual bodies (with respect to the participant's body) may be due to the frequent mirror exposure of humans.

The present experimental data on MA and agency for full-body locomotion and navigation complement recent experimental data (Aspell et al., 2009; Ehrsson, 2007; Lenggenhager, Mouthon, \& Blanke, 2009; Lenggenhager et al., 2007; Petkova \& Ehrsson, 2008) and clinical data (Blanke, Landis, Spinelli, \& Seeck, 2004; Blanke \& Metzinger, 2009) on the related sense of full-body ownership and self-location. Based on these previous data it has been argued that a fundamental aspect of bodily self-consciousness and selfhood is its association with a single, whole body, not with multiple body parts as mostly investigated in studies on agency (as discussed above), but also in studies on body ownership (for discussion see Blanke \& Metzinger, 2009). Thus, a number of recent studies (Aspell et al., 2009; Ehrsson, 2007; Lenggenhager et al., 2009; Lenggenhager et al., 2007; Mizumoto \& Ishikawa, 2005; Petkova \& Ehrsson, 2008) demonstrated that more global aspects of bodily experience (such as full-body body ownership and self-location) can be experimentally manipulated using multisensory conflicts. The present study used sensorimotor conflicts to study full-body agency and therefore complements this previous work on the multisensory aspects of selfhood. We also note that these experimental studies on fullbody ownership and self-location were inspired by an unusual and 
revealing set of neurological phenomena - autoscopic phenomena - in which the sense of the body as a whole can be disrupted in different ways, and which are likely to be caused by an underlying abnormality in the multisensory integration of full-body bodily inputs (Blanke et al., 2004, 2005; Blanke \& Metzinger, 2009). It may be interesting for future studies to investigate how the neurobiological mechanisms of full-body agency and full-body ownership overlap and differ with respect to those concerning body parts such as the upper extremity.

In conclusion, our data reveal the limits of consciousness for the position of the body and locomotion, allowing us to experimentally investigate the mechanisms of selfhood in moving agents. We show that the control of full-body locomotion and the building of a conscious experience of it are at least partially distinct brain processes. Under certain experimental conditions healthy human agents behave "zombie-like" or like sleepwalkers, that is, are not monitoring correctly (or only with low precision) the movement and position of their body in space. This low precision may actually be an advantage in everyday life. William James mentioned that we perform worse when consciously descending a staircase step by step. Similarly, during locomotion humans may not be precisely aware of their location and movement in space so that the brain can deal with a number of situations automatically and often even better without awareness. Only if errors pass a certain threshold - here of $10-15^{\circ}$ - do humans become conscious and walking parameters may be adapted through conscious control. We argue that these mechanisms may be abnormal in patients with somnambulism and epileptic episodic nocturnal wandering and that their further study may help to refine our scientific and legal criteria of selfhood.

\section{Acknowledgements}

The authors would like to thank Dr. Marc Jeannerod and Dr. Chris Frith for comments on a previous version of the manuscript. The authors were supported by the Swiss National Science foundation, the Sandoz Foundation, and the Deutsche Forschungsgemeinschaft.

\section{Appendix A. Supplementary data}

Supplementary data associated with this article can be found, in the online version, at doi:10.1016/j.neuropsychologia.2010.02.005.

\section{References}

Armstrong, D. M. (1988). The supraspinal control of mammalian locomotion. Journal of Physiology, 405, 1-37.

Aspell, J. E., Lenggenhager, B., \& Blanke, O. (2009). Keeping in touch with one's self: Multisensory mechanisms of self-consciousness. PLoS One, 4(8), e6488.

Beran, R. G. (2002). Automatism: Comparison of common law and civil law approaches-a search for the optimal. Journal of Law Medicine, 10(1), 61-68.

Blanke, O., Landis, T., Spinelli, L., \& Seeck, M. (2004). Out-of-body experience and autoscopy of neurological origin. Brain, 127(Pt 2), 243-258.

Blanke, O., \& Metzinger, T. (2009). Full-body illusions and minimal phenomenal selfhood. Trends in Cognitive Science, 13(1), 7-13.

Blanke, O., Mohr, C., Michel, C. M., Pascual-Leone, A., Brugger, P., Seeck, M., et al. (2005). Linking out-of-body experience and self processing to mental own-body imagery at the temporoparietal junction. Journal of Neuroscience, 25(3), 550-557.

Bridgemen, B., Kirch, M., \& Sperling, A. (1981). Segregation of cognitive and motor aspects of visual function using induced motion. Perception in Psychophysics, 29(4), 336-342.

Broughton, R. J. (1968). Sleep disorders: disorders of arousal? Enuresis, somnambulism, and nightmares occur in confusional states of arousal, not in "dreaming sleep". Science, 159(819), 1070-1078.

Castiello, U., Paulignan, Y., \& Jeannerod, M. (1991). Temporal dissociation of motor responses and subjective awareness. A study in normal subjects. Brain, 114(Pt 6), 2639-2655.

Cutting, J. E., Moore, C., \& Morrison, R. (1988). Masking the motions of human gait. Perception in Psychophysics, 44(4), 339-347.

David, N., Bewernick, B. H., Cohen, M. X., Newen, A., Lux, S., Fink, G. R., et al. (2006). Neural representations of self versus other: visual-spatial perspective taking and agency in a virtual ball-tossing game. Journal of Cognitive Neuroscience, 18(6), 898-910.

Drew, T., Prentice, S., \& Schepens, B. (2004). Cortical and brainstem control of locomotion. Progress in Brain Research, 143, 251-261.

Ehrsson, H. H. (2007). The experimental induction of out-of-body experiences. Science, 317(5841), 1048.

Farrer, C., Franck, N., Paillard, J., \& Jeannerod, M. (2003). The role of proprioception in action recognition. Consciousness and Cognition, 12(4), 609-619.

Fourneret, P., Franck, N., Slachevsky, A., \& Jeannerod, M. (2001). Self-monitoring in schizophrenia revisited. Neuroreport, 12(6), 1203-1208.

Fourneret, P., \& Jeannerod, M. (1998). Limited conscious monitoring of motor performance in normal subjects. Neuropsychologia, 36(11), 1133-1140.

Franck, N., Farrer, C., Georgieff, N., Marie-Cardine, M., Dalery, J., d'Amato, T., et al. (2001). Defective recognition of one's own actions in patients with schizophrenia. American Journal of Psychiatry, 158(3), 454-459.

Fukuyama, H., Ouchi, Y., Matsuzaki, S., Nagahama, Y., Yamauchi, H., Ogawa, M., et al. (1997). Brain functional activity during gait in normal subjects: a SPECT study. Neuroscience Letters, 228(3), 183-186.

Gastaut, H., \& Broughton, R. (1965). A clinical and polygraphic study of episodic phenomena during sleep. In J. Wortis (Ed.), Recent advances in biological psychiatry (pp. 197-221). New York: Plenum Press.

Goodale, M. A., Milner, A. D., Jakobson, L. S., \& Carey, D. P. (1991). A neurological dissociation between perceiving objects and grasping them. Nature, 349(6305), 154-156.

Goodale, M. A., Pelisson, D., \& Prablanc, C. (1986). Large adjustments in visually guided reaching do not depend on vision of the hand or perception of target displacement. Nature, 320(6064), 748-750.

Grillner, S., \& Wallen, P. (1985). Central pattern generators for locomotion, with special reference to vertebrates. Annual Review of Neuroscience, 8, 233-261.

Hicheur, H., Pham, Q. C., Arechavaleta, G., Laumond, J. P., \& Berthoz, A. (2007). The formation of trajectories during goal-oriented locomotion in humans. I: A stereotyped behaviour. European Journal of Neuroscience, 26(8), 2376-2390.

Jeannerod, M., \& Pacherie, E. (2004). Agency, simulation and self-identification. Mind E Language, 19(2), 34.

Knoblich, G., \& Kircher, T. T. (2004). Deceiving oneself about being in control: conscious detection of changes in visuomotor coupling. Journal of Experimental Psychology: Human Perception and Performance, 30(4), 657-666.

Knoblich, G., Stottmeister, F., \& Kircher, T. (2004). Self-monitoring in patients with schizophrenia. Psychological Medicine, 34(8), 1561-1569.

Koch, C., \& Crick, F. (2001). The zombie within. Nature, 411(6840), 893.

Lenggenhager, B., Mouthon, M., \& Blanke, O. (2009). Spatial aspects of bodily selfconsciousness. Consciousness and Cognition, 18(1), 110-117.

Lenggenhager, B., Tadi, T., Metzinger, T., \& Blanke, O. (2007). Video ergo sum: manipulating bodily self-consciousness. Science, 317(5841), 1096-1099.

Mahowald, M. W., Bundlie, S. R., Hurwitz, T. D., \& Schenck, C. H. (1990). Sleep violence-forensic science implications: polygraphic and video documentation. Journal of Forensic Science, 35(2), 413-432.

Miyai, I., Tanabe, H. C., Sase, I., Eda, H., Oda, I., Konishi, I., et al. (2001). Cortical mapping of gait in humans: A near-infrared spectroscopic topography study. Neuroimage, 14(5), 1186-1192.

Mizumoto, M., \& Ishikawa, M. (2005). Immunity to error through misidentification and the bodily illusion experiment. Journal of Consciousness Studies, 12(7), 17.

Nielsen, T. (1963). Volition: A new experimental approach. The Scandinavian Journal of Psychology, 4, 6.

Nielsen, T. (1978). Acts. Analysis and synthesis of human acting, concerning the subject and from the standpoint of the subject. Copenhagen: Dansk Psykologisc Forlag.

Nutt, J. G., Marsden, C. D., \& Thompson, P. D. (1993). Human walking and higher-level gait disorders, particularly in the elderly. Neurology, 43(2), 268-279.

Pedley, T. A., \& Guilleminault, C. (1977). Episodic nocturnal wanderings responsive to anticonvulsant drug therapy. Annals of Neurology, 2(1), 30-35.

Petkova, V. I., \& Ehrsson, H. H. (2008). If I were you: Perceptual illusion of body swapping. PLoS One, 3(12), e3832.

Plazzi, G., Tinuper, P., Montagna, P., Provini, F., \& Lugaresi, E. (1995). Epileptic nocturnal wanderings. Sleep, 18(9), 749-756.

Posada, A., Franck, N., Augier, S., Georgieff, N., \& Jeannerod, M. (2007). Altered processing of sensorimotor feedback in schizophrenia. C R Biology, 330(5), 382-388.

Razzaque, S., Kohn, Z., \& Whitton, M. C. (2001). Redirected Walking. Paper presented at the Eurographics 2001.

Reed, C. L., Stone, V. E., Bozova, S., \& Tanaka, J. (2003). The body-inversion effect. Psychological Science, 14(4), 302-308.

Revonsuo, A., Johanson, M., Wedlund, J., \& Chaplin, J. (2000). The zombies among us: Consciousness and automatic behaviour. In Y. Rossetti \& A. Revonsuo (Eds.), Beyond Dissociation Interaction between dissociated implicit and explicit processing (p. 372). Amsterdam: John Benjamins Publishing Company.

Slachevsky, A., Pillon, B., Fourneret, P., Pradat-Diehl, P., Jeannerod, M., \& Dubois, B. (2001). Preserved adjustment but impaired awareness in a sensory-motor conflict following prefrontal lesions. Journal of Cognitive Neuroscience, 13(3), 332-340.

Suzuki, M., Miyai, I., Ono, T., Oda, I., Konishi, I., Kochiyama, T., et al. (2004). Prefrontal and premotor cortices are involved in adapting walking and running speed on the treadmill: An optical imaging study. Neuroimage, 23(3), 1020-1026. 
Synofzik, M., Thier, P., \& Lindner, A. (2006). Internalizing agency of self-action: perception of one's own hand movements depends on an adaptable prediction about the sensory action outcome. Journal of Neurophysiology, 96(3), 1592-1601.

Tsakiris, M., \& Haggard, P. (2005). The rubber hand illusion revisited: Visuotactile integration and self-attribution. Journal of Experimental Psychology: Human Perception and Performance, 31(1), 80-91. van den Bos, E., \& Jeannerod, M. (2002). Sense of body and sense of action both contribute to self-recognition. Cognition, 85(2), 177-187.

Vogeley, K., \& Fink, G. R. (2003). Neural correlates of the first-person-perspective. Trends in Cognitive Science, 7(1), 38-42. 